\title{
Bulliyng e o comportamento de jovens de escolas de ensino fundamental
}

\section{Bullying and behavior of scholchildren from elementary school}

\author{
Gehysa Guimarães Alves, ${ }^{1}$ Guilherme Anziliero Arossi, ${ }^{1}$ Marina Flamia Haas, ${ }^{1}$ Ana Maria Pujol Vieira \\ dos Santos, ${ }^{1}$ Maximiano Ferreira Tovo ${ }^{1}$ \\ Universidade Luterana do Brasil (ULBRA), Torres, RS, Brasil.
}

Recebido em: 21/08/2016 / Aceito em: 26/09/2016 / Publicado em: 18/10/2016

guilhermeclinica@gmail.com

\section{RESUMO}

Bullying é um termo utilizado para descrever atos de violência física ou psicológica, intencionais e repetidos, praticados por um indivíduo ou um grupo de indivíduos, com o propósito de intimidar ou agredir o outro para satisfação pessoal e demonstração de poder. Objetivo: descrever a frequência de bullying e seus fatores associados. Método: estudo descritivo de série de casos no qual a população estudada foram alunos do sexto ao nono ano do ensino fundamental de cinco escolas gaúchas da Rede ULBRA de ensino fundamental. Para a coleta dos dados, foram utilizados questionários autoaplicáveis, de modo a caracterizar comportamento, idade e série escolar. Além disso, foi aplicado o questionário Kidscape, com perguntas objetivas relacionadas ao bullying e o quanto este interfere em suas vidas. Os dados quantitativos foram analisados com estatística descritiva. Para a análise dos dados qualitativos, foi utilizada a análise de conteúdo temática. Resultados: $34 \%$ já haviam sofrido Bullying e $17 \%$ afirmaram já ter agredido ou intimidado alguém. Na questão, "se sentem felizes", 84,9\% dos entrevistados referiram que sim. Apenas $13,2 \%$ referiu que se sentiam menos ou muito menos que de costume. Para os jovens, a felicidade está relacionada a ter família, amigos, lazer e viver bem a vida. Sobre ter perdido a confiança em si próprio, percentual aproximado de alunos respondeu que sim $(9,4 \%)$; também referiram que "sentem-se pouco capazes de resolver problemas". 66\% referiram "sentir prazer nas atividades que realizam," e 24,5\% referiram "sentir-se sob pressão em seu dia a dia". Além disso, 22,6\% apontaram que conseguem se "concentrar menos no último ano", 18,9\% perde "sono com preocupações". Considerações finais: conclui-se que o bullying é um fenômeno complexo, interdisciplinar e está relacionado com a autoconfiança, com as relações familiares e com sentir-se feliz.

Palavras-chave: Bullying; Violência; Saúde Escolar.

\section{ABSTRACT}

Bullying is a term used to describe acts of physical or psychological violence, intentional, repeated, and performed by an individual or a group of individuals with the purpose of intimidating or harming the other for personal satisfaction and demonstration of power. Objective: to describe the frequency of bullying and its associated factors. Method: descriptive study of case series in which the population studied were students from the sixth to ninth grade of five private elementary school from Southern Brazil. Data were collected using self-administered questionnaires to characterize behavior, age and grade. In addition we applied the questionnaire Kidscape with objective questions related to bullying and how this interferes with their lives. Quantitative data were analyzed with descriptive statistics. For the analysis of qualitative data we used the thematic content analysis. Results: $34 \%$ had experienced bullying and $17 \%$ said they had assaulted or bullied someone. In the question "do you feel happy" $84.9 \%$ of respondents said yes. Only 13.2\% reported that they felt less or much less than usual. For young people happiness is related to be family, friends, leisure and living life well. About having lost confidence in himself significant percentage of students answered yes (9.4\%); also they said it "feel barely able to solve problems." 66\% said they "feel pleasure in activities they perform," and $24.5 \%$ said they "feel under pressure in their daily lives." In addition, $22.6 \%$ said they are able to "focus less on last year", 18.9\% lost "sleep with concerns." Closing remarks: it is concluded that bullying is a complex, interdisciplinary phenomenon and is related to the self-confidence, famiIy relationships and feel happy.

Keywords: Bullying; Violence; School Health. 


\section{INTRODUÇÃO}

A agressividade nas escolas é um problema universal. O bullying - termo de origem inglesa ${ }^{1}$ - representa um tipo de violência que ocorre durante a infância e a adolescência. ${ }^{2}$ Compreende todas as condutas de agressão/vitimização que ocorrem entre pares, de forma intencional e repetida, sem motivação evidente, adotada por um ou mais estudantes contra outro(s), na qual há o abuso de alguém mais forte, para com alguém mais fraco, causando dor e angústia. Assim, o bullying é executado dentro de uma relação desigual de poder. ${ }^{1,3,4}$ Este fenômeno começou a ser estudado na Suécia, na década de 1970. No Brasil, na década de 1990, o bullying passou a ser discutido. No entanto, foi a partir de 2005 que o tema passou a ser objeto de reflexão e pesquisa e seus resultados divulgados em artigos científicos. Embora os estudos sobre o bullying escolar no Brasil sejam recentes, o fenômeno é antigo e preocupante, sobretudo em função de seus efeitos nocivos. Em muitos países, o bullying nas escolas e a violência juvenil tornaram-se grandes preocupações. Incidentes como o massacre em Erfurt, Alemanha, em 2002, no qual 18 pessoas foram mortas e os suicídios de adolescentes, devido ao assédio moral, na Escócia, em 2002, têm aumentado a conscientização pública sobre estas questões.

Ele pode se apresentar na forma de uma agressão física, verbal ou pode incluir outros comportamentos, objetivando manter o outro fora do grupo. Atualmente, há fenômenos mais recentes como o Cyberbullying, que se caracteriza por enviar mensagens pejorativas por e-mail ou telefone móvel, colocar/enviar imagens embaraçosas sobre uma pessoa nas redes sociais, usar o nome de outra pessoa para espalhar mentiras na Internet. ${ }^{4-8}$

$\mathrm{Na}$ prática de Bullying há um padrão repetitivo de comportamento que visa lesar alguém, procurando manter um desequilíbrio de poder. $O$ agressor sente satisfação em magoar repetidamente o seu alvo, pois assim constrói a sua própria noção de poder, acreditando que o alvo é mais fraco física, mental e/ou emocionalmente. ${ }^{6,8}$ Pode ser cometido por um ou vários estudantes que agem contra uma única vítima. ${ }^{4,6}$

Fatores econômicos, sociais e culturais, aspectos inatos de temperamento e influências familiares, de amigos, da escola e da comunidade, constituem riscos para a manifestação do bullying, que pode causar impacto na saúde e desenvolvimento de crianças e adolescentes. ${ }^{1}$ Existem três elementos que caracterizam o bullying: repetição, prejuízo e desigualdade de poder. ${ }^{6}$ No que diz respeito à sua personalidade, as vítimas tendem a ser ansiosas e inseguras, com baixa autoestima e têm tendências depressivas que persistem na adolescência e início da idade adulta, mesmo depois que a agressão tenha parado. Isso pode conduzir ao insucesso na escola e sintomas psicossomáticos. ${ }^{7,9}$ Quanto ao agressor, este se caracteriza por ser tipicamente popular, tende a envolver-se em uma variedade de comportamentos antissociais, tem características impulsivas e geralmente entende sua própria agressividade como qualidade, é geralmente mais forte que seu alvo, sente prazer e satisfação em dominar, controlar e causar danos e sofrimentos a outros., As vítimas de bullying geralmente sentem-se feri- das, assustadas, sozinhas e tristes, percebendo a escola como um lugar de medo, estresse e violência. ${ }^{8}$ As testemunhas são aqueles que não se envolvem diretamente em Bullying, mas são envolvidos como espectadores. A maioria não consegue apoiar ou auxiliar a vítima por não saber o que fazer, por ter medo de se tornar a próxima vítima ou por medo de fazer algo errado e causar problemas ainda maiores. ${ }^{6}$ A presença de público, com frequência, incentiva os ataques. ${ }^{10}$

Os efeitos negativos do bullying não afetam apenas a vítima, mas também a família, a escola e até mesmo a sociedade, uma vez que estudos longitudinais têm demonstrado que crianças que são maltratadas são mais propensas a desenvolver comportamento antissocial, como o vandalismo, abuso de drogas e ataques violentos na vida adulta, ${ }^{11-13}$ bem como baixa autoestima e menos empatia com outras pessoas. ${ }^{14}$ No entanto, também afetam as testemunhas que os agressores, pois apontam para uma maneira de viver a vida.

A escola, por sua vez, proporciona a experiência de relações de hierarquia e convívio com as diferenças. É um local de aprendizagem, no qual são estabelecidas ordens e normas para que os alunos aprendam e auxiliam na formação do indivíduo. ${ }^{4,6}$ No entanto, em função de concentrar muitas crianças e adolescentes, também é um território propício à prática do Bullying. Contudo, essa prática não está restrita à escola, podendo surgir também em outros espaços, tais como o percurso para a escola, campos de férias, clubes desportivos, no ciberespaço, entre outros. Considerando-se que a maioria dos atos de bullying ocorre fora da visão dos adultos, que grande parte das vítimas não reage ou fala sobre a agressão sofrida, pode-se entender porque professores e pais têm pouca percepção do bullying, subestimam a sua prevalência e atuam de forma insuficiente para a redução e interrupção dessas situações. ${ }^{1,5}$ Quando há consciência dos professores e pais sobre a existência do bullying, o ambiente escolar consegue ser mais protetor, uma vez que os professores se empenham em tentar combatê-lo. ${ }^{5}$

Em função da importância do tema, este trabalho objetiva descrever a frequência de bullying (violência escolar) e seus fatores associados, em adolescentes de ensino fundamental.

\section{MÉTODO}

Este é um estudo descritivo que envolve 53 alunos com idades entre 12 a 15 anos, de ambos os sexos, do sexto ao nono ano, do ensino fundamental, de cinco escolas gaúchas privadas de ensino fundamental.

A equipe de pesquisa visitou as escolas e, após consentimento da direção, entregou para os alunos que poderiam fazer parte do estudo o Termo de Consentimento Livre e Esclarecido (TCLE), para que seus pais assinassem. Foi combinado o dia de retorno para a realização da entrevista. Assim, foram incluídos no estudo todos os alunos que estavam presentes na sala de aula no dia da coleta de dados e que tinham levado o TCLE, assinado pelos responsáveis.

Para a coleta dos dados, foram utilizados questionários autoaplicáveis, que levantaram variáveis sobre 
comportamento violento, percepção de felicidade, idade e série escolar. Além disso, foi aplicado o questionário Kidscape com perguntas objetivas relacionadas ao bullying (www.kidscape.org.uk), e o quanto este interfere em suas vidas (transcendência do bullying).

Os instrumentos de coleta de dados foram digitalizados utilizando o software Epidata 3.1. e os dados quantitativos foram analisados através do software de análise estatística SPSS 17.0 for Windows. A análise dos dados quantitativos foi realizada por estatística descritiva. Em relação aos dados qualitativos, foi utilizada a análise de conteúdo temática.

Em relação aos aspectos éticos, as escolas participantes autorizaram a realização da pesquisa e o projeto foi aprovado pelo Comitê de Ética em Pesquisa em Seres Humanos da ULBRA (CEP/ULBRA), sob o parecer 891.311.

\section{RESULTADOS}

Dos 53 alunos investigados, a grande maioria tinha 13 ou 14 anos, perfazendo um total de 75,5\% (Tabela 1). Quando perguntados se já haviam sofrido Bullying em algum momento de sua vida, 34\% referiu que sim e nove alunos $(17 \%)$ afirmaram já ter agredido ou intimidado alguém (Tabela 2).

Tabela 1 - Idade dos alunos participantes da pesquisa.

\begin{tabular}{lcc}
\hline Idade em anos & Frequência & $\%$ \\
\hline 12,00 & 2 & 3,8 \\
13,00 & 17 & 32,1 \\
14,00 & 23 & 43,4 \\
15,00 & 11 & 20,8 \\
Total & 53 & 100,0 \\
\hline Média & 13,8 anos & \\
\hline
\end{tabular}

Tabela 2 - Ocorrência de bullying na escola.

\begin{tabular}{lccc}
\hline Já sofreu bullying & Frequência & $\%$ & $\%$ válida \\
\hline Não & 33 & 62,3 & 63,5 \\
Sim & 18 & 34,0 & 34,6 \\
Total & 52 & 98,1 & 100 \\
Ausente & 1 & 2,9 & \\
Total & 53 & 100 & \\
\hline Agressor. Você já & & & \\
agrediu, intimidou? & & & \\
\hline Sim & 9 & 17,0 & 17,3 \\
Não & 43 & 81,1 & 82,7 \\
Total & 52 & 98,1 & 100 \\
Ausente & 1 & 1,9 & \\
Total & 53 & 100 & \\
\hline
\end{tabular}

Ao serem questionados sobre sentimento de felicidade, $84,9 \%$ dos entrevistados referiram que se sentem felizes. Apenas $13,2 \%$ referiu que se sentia menos ou muito menos que de costume. Sobre ter perdido a confiança em si próprio, praticamente o mesmo percentual de alunos que respondeu que $\operatorname{sim}(9,4 \%)$, também referiram que se sentem pouco capazes de resolver problemas. Ao serem questionados sobre sua participação em brigas, 18,9\% referiram ter participado em, no mínimo, uma no último ano. No entanto, sobre sentirem prazer nas atividades que realizam, este percentual sobe para $66 \%$ e $24,5 \%$ referiram sentir-se sob pressão em seu dia a dia. Além disso, 22,6\% apontaram que conseguem se concentrar menos no último ano, 18,9\% perde sono com preocupações (Tabela 3).

Tabela 3 - Descrição dos resultados obtidos.

\begin{tabular}{|c|c|c|c|}
\hline Sente-se feliz? & Frequência & $\%$ & \% válida \\
\hline Mais que o de costume & 27 & 50,9 & 51,9 \\
\hline O mesmo que o de costume & 18 & 34,0 & 34,6 \\
\hline Menos que o de costume & 3 & 5,7 & 5,8 \\
\hline Muito menos que o de costume & 4 & 7,5 & 7,7 \\
\hline Total & 52 & 98,1 & 100,0 \\
\hline Ausente & 1 & 1,9 & \\
\hline Total & 53 & 100,0 & \\
\hline \multicolumn{4}{|l|}{$\begin{array}{l}\text { Tem perdido a confiança em } \\
\text { você? }\end{array}$} \\
\hline Não & 39 & 73,6 & 76,5 \\
\hline Não mais que o de costume & 3 & 5,7 & 5,9 \\
\hline Mais que o de costume & 4 & 7,5 & 7,8 \\
\hline Muito mais que o de costume & 5 & 9,4 & 9,8 \\
\hline Total & 51 & 96,2 & 100,0 \\
\hline Ausente & 2 & 3,8 & \\
\hline Total & 53 & 100,0 & \\
\hline \multicolumn{4}{|l|}{ É capaz de enfrentar problemas? } \\
\hline Mais que o de costume & 22 & 41,5 & 43,1 \\
\hline O mesmo que o de costume & 19 & 35,8 & 37,3 \\
\hline Menos que o de costume & 5 & 9,4 & 9,8 \\
\hline Muito menos que o de costume & 5 & 9,4 & 9,8 \\
\hline Total & 51 & 96,2 & 100,0 \\
\hline Ausente & 2 & 3,8 & \\
\hline Total & 53 & 100,0 & \\
\hline
\end{tabular}

\section{Sente prazer nas atividades}

que faz?

\begin{tabular}{|c|c|c|c|}
\hline Mais que o de costume & 15 & 28,3 & 31,3 \\
\hline O mesmo que o de costume & 20 & 37,7 & 41,7 \\
\hline Menos que o de costume & 9 & 17,0 & 18,8 \\
\hline Muito menos que o de costume & 4 & 7,5 & 8,3 \\
\hline Total & 48 & 90,6 & 100,0 \\
\hline Ausente & 5 & 9,4 & \\
\hline Total & 53 & 100,0 & \\
\hline \multicolumn{4}{|l|}{ Sente-se sob pressão? } \\
\hline Não & 26 & 49,1 & 52,0 \\
\hline Não mais que o de costume & 11 & 20,8 & 22,0 \\
\hline Mais que o de costume & 9 & 17,0 & 18,0 \\
\hline Muito mais que o de costume & 4 & 7,5 & 8,0 \\
\hline Total & 50 & 94,3 & 100,0 \\
\hline Ausente & 3 & 5,7 & \\
\hline Total & 53 & 100,0 & \\
\hline \multicolumn{4}{|l|}{ Perdeu sono com preocupação? } \\
\hline Não & 31 & 58,5 & 59,6 \\
\hline Não mais que o costume & 11 & 20,8 & 21,2 \\
\hline Mais que o costume & 8 & 15,1 & 15,4 \\
\hline Total & 52 & 3,8 & 3,8 \\
\hline Ausente & 1 & 98,1 & 100,0 \\
\hline Total & 53 & 100,0 & \\
\hline \multicolumn{4}{|l|}{ Consegue se concentrar? } \\
\hline Mais que o de costume & 15 & 28,3 & 28,8 \\
\hline O mesmo que o costume & 25 & 47,2 & 48,1 \\
\hline Menos que o costume & 12 & 22,6 & 23,1 \\
\hline Total & 52 & 98,1 & 100,0 \\
\hline Ausente & 1 & 1,9 & \\
\hline Total & 53 & 100,0 & \\
\hline
\end{tabular}


Em informações adicionais, os adolescentes, ao falar sobre o que entendem por felicidade, referiram que é sentir-se bem, estar bem consigo e com a vida. As questões mais apontadas pelos escolares foram ter amigos e família, diversão, amor e dinheiro, fazendo aquilo que se gosta com as pessoas que se ama. Discorreram sobre qualidade de vida possibilitada pelo dinheiro, que ajuda a ter comida e adquirir objetos novos. No entanto, é preciso ter educação, segurança, dormir bem e estar de bem com a vida. Salientaram também sobre a importância de se ter paz, saúde, respeito, tranquilidade e liberdade, praticar esportes, ajudar os outros, agir em busca dos seus sonhos e aproveitar cada minuto de seu viver.

\section{DISCUS5ÃO}

$\mathrm{Na}$ literatura encontram-se estudos que identificaram cerca de $30 \%$ de ocorrência de Bullying entre alunos do ensino fundamental, ${ }^{15,16}$ dado semelhante ao encontrado em nossa pesquisa (34\%). Os achados de Moura et al. (2011) ${ }^{17}$ referiram valores menores, cerca de 17\%, em alunos de Pelotas. Em Porto Alegre, no estudo de Bandeira e Hutz, ${ }^{6}$ a prevalência de vítimas de Bullying foi de $23 \%$, enquanto que em Grossi e Santos ${ }^{18}$ foi de $70 \%$, como vítimas ou agressores. Essa variabilidade nos dados de prevalência pode ser explicada pela limitação metodológica que os instrumentos de coleta de dados utilizados apresentam, que vão desde a heterogeneidade dos instrumentos, até o próprio conceito de Bullying. ${ }^{19}$ Em revisão sistemática da literatura, a variabilidade da prevalência de Bullying encontrada no Brasil, se situa entre $10,2 \%$ a $31,1 \% .{ }^{20}$

A Tabela 3 mostra que 9,4\% dos participantes da pesquisa haviam perdido a confiança em si próprio, praticamente o mesmo percentual de alunos que referiram sentir-se pouco capazes de resolver problemas. Considerando que cerca de $30 \%$ dos alunos sofrem Bullying e apenas cerca de $10 \%$ relatam ter perdido a autoconfiança e se julgarem incapazes de resolver problemas, percebe-se que uma parcela destes alunos consegue lidar com a situação estressora do Bullying. Isso acaba influenciando significativamente o dia-a-dia dos que não conseguem lidar com este problema, o que pode ser explicado em função dos mecanismos de resiliência que cada jovem desenvolve, ajudando-os a lidar com esse tipo de violência. $O$ estudo realizado por Bandeira e Hutz, ${ }^{6}$ constatou que das vítimas de Bullying, 31,8\% não se incomodaram com as atitudes dos colegas. A resiliência que cada um possui é um fator fundamental para que esses alunos consigam superar as possíveis consequências do Bullying. ${ }^{21}$ Isso é importante, pois as vítimas se tornam cidadãos estressados, deprimidos, com baixa autoestima, podendo ocasionar doenças psicossomáticas e transtornos mentais. Isso indica a necessidade de programas de saúde do escolar, nos quais se busca a reflexão crítica desses jovens sobre os malefícios da violência, formas de preveni-la e a discussão sobre a cultura da paz.

Segundo Medeiros, Zequinão e Cardoso, ${ }^{22}$ entre as características que tornam o indivíduo mais propenso a ser vítima de Bullying está a demonstração de problemas de coordenação motora, estando estes indivíduos mais predispostos a vulneráveis intimidações, tornando-os víti- mas frequentes. Esse fato contribui para a redução da prática de atividades físicas, bem como a menor frequência às aulas de Educação Física escolar, o que contribui para maiores riscos à saúde desses indivíduos vitimados.

Em nosso estudo, 24,5\% referiram sentir-se sob pressão. Estudo realizado salienta que o Bullying desperta em suas vítimas sentimentos de raiva e medo, levando a vítima a ficar assustada (38\%) e preocupada $(24 \%)^{18}$. Dessa forma, mesmo que a vítima desenvolva mecanismos de resiliência e mantenha sua autoconfiança e capacidade de resolver problemas, a violência sofrida contribui para criar um ambiente não-saudável dentro do ambiente escolar, com aproximadamente um terço dos alunos, sentindo-se sob pressão.

No que diz respeito aos agressores, o resultado aqui apresentado mostra que $17 \%$ dos participantes afirmaram já ter agredido ou intimidado alguém, dado semelhante aos encontrados por Bandeira e Hutz ${ }^{6}$ $15,9 \%$ - e por Calbo et al. ${ }^{23}$ - 12,58\%. Os meninos são a maioria dos agressores, numa proporção de dois agressores meninos a cada menina praticante de Bullying, o que pode ser observado, tanto em nosso estudo, quanto na literatura. ${ }^{20,23}$

Felicidade ou bem-estar subjetivo é o termo utilizado para referir-se ao pensamento ou sentimento dos indivíduos em relação às suas vidas. Por isso, se utiliza o termo subjetivo, já que fala sobre uma avaliação da qualidade de vida de cada sujeito e leva em consideração questões como seu trabalho, família e vida social, como é influenciado pelas emoções e humores experimentados no cotidiano. É, portanto, tanto uma avaliação cognitiva, como emocional. ${ }^{24}$

A percepção de felicidade expressa pelos escolares aponta esta avaliação da vida como algo externo a si próprio, pois a coloca na dependência de ter ou alcançar algo. Assim, fica condiciona ao "se .... então serei feliz". Quando a pessoa acredita que a felicidade tem relação com o ter ou com o se....então, ela se coloca como expectadora de sua própria vida, idealizando este estado e se afastando do que é possível construir para si próprio. ${ }^{25}$ Três aspectos são importantes quando se fala em felicidade ou bem estar subjetivo. São eles: este sentimento nem sempre é afetado por condições materiais, pois depende de valores individuais; ele tem sido considerado como resultado do balanço entre afetos positivos e negativos; e inclui o julgamento dos aspectos que envolvem a vida de cada um. ${ }^{26}$ Quando se olha para a fala desses escolares, o que se percebe é que as condições materiais são muito importantes na vida desses adolescentes. É possível que isso ocorra em função de que não tenham condições muito favoráveis de vida, tendo o desejo de adquirir objetos, viajar, sair e se divertir e não conseguir em função de sua condição econômica e de sua falta de autonomia financeira, já que o dinheiro vem do trabalho da família e eles, em função da idade, ainda são muito dependentes. Contudo, não foi encontrada relação entre Bullying e condição socioeconômica, com sua ocorrência permeando as diferentes classes sociais. ${ }^{27}$

Esta percepção reforça a ideia de que a experiência de bem estar é interna a cada sujeito, sendo suas crenças e a maneira como vê a sua vida de fundamental importância para esta percepção. A proximidade com a 
família pode ser considerado como fator protetor já que quando as relações são positivas propiciam apoio emocional. ${ }^{24}$ Isto pode estar relacionado com a fala de grande parte dos adolescentes entrevistados que apontou a família como o principal fator. Na vida dos jovens, esses fatores determinam o estado de bem estar juntamente com a satisfação dos desejos, lazer e paz, referidos de alguma forma por esses escolares. ${ }^{28}$

Em estudo realizado, semelhante ao nosso, família, amizades, relacionamentos, sentimentos de paz, carinho, liberdade e segurança, atividades de lazer e satisfação com as necessidades materiais foram referidas pelos adolescentes entrevistados. ${ }^{24}$ Percebe-se uma tendência dos jovens de relacionar a felicidade com sentir-se bem consigo próprio e com os outros e com ter e estar bem com sua família. Esta última pode ser considerada uma rede de apoio importante para este jovem reforçar sentimentos positivos frente a sua vida. Isto auxilia a pessoa a se conectar mais com sua potência, conhecer melhor seus desejos e agir na direção de sua realização.

\section{CONSIDERACִÕES FINAIS}

Apesar da amostra deste estudo ser relativamente pequena, seus achados apontam para a importância de se desenvolver ações que reduzam as situações de violência dentro da escola, envolvendo não somente professores e alunos, mas toda a comunidade escolar: funcionários, direção, pais e moradores do entorno da escola. Situações de violência não devem ser toleradas sob pena de estarem reforçando relações de poder desiguais e contribuindo para uma sociedade excludente e violenta. É preciso discutir questões que apontem para uma sociedade mais justa, mais solidaria e mais democrática, na qual a cultura da paz permeie as relações que se estabelecem dentro e fora do ambiente escolar.

\section{REFERÊNCIAS}

1. Lopes Neto Aramis A. Bullying: comportamento agressivo entre estudantes. J Pediatr 2005;81(5):164-172. doi: 10.1590/S0021-7557200500070000

2. Craig WM, Harel Y. Bullying, physical fighting and victimization. In: Currie C, Roberts C, Morgan A, Smith R, Settertobulte W, Samdal O, et al. (editors). Young peoples health in context. Health Behavior in School-aged Children (HBSC) study: international report from the 2001/2002 survey. Health Policy for Children and Adolescents. World Health Organization 2004;(4):133-144.

3. Pearce JB, Thompson AC. Practical approaches to reduce the impact of bullying. Arch Dis Child 1998;79(6):528-31. doi: 10.1136/adc.79.6.528.

4. Cavalcanti AL. Lesões no complexo maxilofacial em vítimas de violência no ambiente escolar. Cien Saude Colet 2009;14(5):1835-1842.

5. Fekkes M, Pijpers FI, Verloove-Vanhorick SP. Bullying: Who does what, when and where? Involvement of children, teachers and parents in bullying behavior. Health Educ Res.2005;20(1):81-91. doi: 10.1093/her/cyg100.

6. Bandeira CdeM, Hutz CS. Bullying: prevalência, implicações e diferenças entre os gêneros. Psicol Esc Educ 2012;16(1):35-44.

7. Olweus D. Victimisation by peers: antecedents and long term outcomes. In: Rubin $\mathrm{KH}$, Asendorpf JB, eds. Social Withdrawal, Inhibition and Shyness in Childhood. Hillsdale, NJ: Lawrence Erlbaum Associates 1993;315-341.

8. Castro DSOS, Silva NMRB. Bullying: uma outra guerra. Rev Milit 2010;(2501/2502):733-743.

9. DiBiase AT, Sandler PJ. Malocclusion, orthodontics and bullying. Dent Update Lon 2001;28(9):464-467.

10. Frick LT. As Relações Entre os Conflitos Interpessoais e o Bullying: Um estudo nos anos iniciais do Ensino Fundamental de duas Escolas. Públicas Presidente Prudente [SP]: Universidade Estadual Paulista, 2011.

11. Bond L, Carlin JB, Thomas L, Rubin K, Patton G. Does bullying cause emotional problems? A prospective study of young teenagers. BMJ 2001;323:480-4. doi: 10.1136/ bmj.323.7311.480.

12. Bender D, Lösel F. Bullying at school as a predictor of delinquency, violence and other anti-social behaviour in adulthood. Crim Behav Ment Health 2011;(21):99-106. doi: 10.1002/cbm.799.

13. Vieno A, Gini G, Santinello M. Different forms of bullying and their association to smoking and drinking behavior in Italian adolescents. J Sch Health 2011;81:393-9. doi: 10.1111/j.1746-1561.2011.00607.x.

14. Olweus D, Limber S, Mihalic SF. Blueprints for Violence Prevention, Book Nine: Bullying Prevention Program. Boulder, CO: Center for the Study and Prevention of Violence; 2002. $109 \mathrm{p}$.

15. Malta DC, Silva MAI, Mello FCMD, Monteiro RA, Sardinha LMV, Crespo C, Porto DL. Bullying nas escolas brasileiras: resultados da Pesquisa Nacional de Saúde do Escolar (PeNSE), 2009. Cien Saude Colet 2010;15(2):3065-3076. doi: 10.1590/S1413-81232010000800011.

16. Do Nascimento KB, de Rosso Krug M, da Costa FTL, do Nascimento BB. Bullying entre escolares: Um estudo descritivo na cidade de cruz alta/rs. Reflexão e Ação 2013;21(1):196-218. doi: 10.17058/rea.v21i1.3418.

17. Moura DR, Cruz ACN, De Ávila Quevedo L. Prevalência e características de escolares vítimas de bullying. J Pediatr 2011;87(1):19-23. doi: 10.2223/JPED. 2042.

18. Grossi PK, Santos AM dos. Desvendando o fenômeno bullying nas escolas públicas de Porto Alegre, RS, Brazil. Rev Portug Educ 2009;22(2):249-267.

19. Vessey J, Strout TD, DiFazio RL, Walker A. Measuring the youth bullying experience: A systematic review of the psychometric properties of available instruments. J Sch Health 2014;84(12):819-843. doi: 10.1111/josh.12210.

20. Nesello F, Sant'Anna FL, Santos HG, Andrade SM, Mesas $A E$, González AD. Características da violência escolar no Brasil: revisão sistemática de estudos quantitativos. Rev Bras Saúde Mater Infant 2014;14(2):119-36. doi: 10.1590/S151938292014000200002.

21. Souza AP. Bullying escolar - uma realidade ainda desconhecida [monografia de Graduação]. Brasília: Centro Universitário do Distrito Federal, 2008.

22. Medeiros $P$, Zequinão $M A$, Cardoso $F L$. Bullying e a relação entre atividade física e coordenação motora: uma revisão sistemática. Cinergis 2014;15(3):158-162.

23. Calbo AS, Busnello FB, Rigoli MM, Schaefer LS, Kristensen $\mathrm{CH}$. Bullying at school: aggressive behavior, victimization and prosocial behavior among peers. Contextos Clinic 2009;2: 73-80. doi: 10.4013/ctc.2009.22.01.

24. Camargo S, Abaid JLW, Giacomoni CH. Do que eles precisam para serem felizes? A felicidade na visão de adolescentes. Rev 
ABRAPE 2011;15(2):241-250.

25. Aerts D, Ganzo C. A felicidade possível. Porto Alegre. Bororó 2011. $25 \mathrm{p}$

26. Freire SA. Bem-estar subjetivo e metas de vida: um estudo transversal com homens e mulheres pertencentes a três faixas de idade [tese]. São Paulo: Universidade Estadual de Campinas, 2011.
27. Tippett N, Wolke D. Socioeconomic status and bullying: a meta-analysis. Amer j pub health 2014;104(6):48-59. doi: 10.2105/AJPH.2014.301960.

28. Giacomoni $\mathrm{CH}$, Hutz CS. Escala multidimensional de satisfação da vida para crianças: estudos de construção e validação. Estud psicol. Campinas 2008;25(1):23-35. doi: 10.1590/S0103-166X2008000100003.

Como citar: AROSSI, Guilherme Anziliero et al. Bulliyng e o comportamento de jovens de escolas de ensino fundamental. Cinergis, Santa Cruz do Sul, v. 18, n. 1, out. 2016. ISSN 2177-4005. Disponível em: <https://online.unisc.br/seer/index.php/ cinergis/article/view/8160>. Acesso em: 11 out. 2016. doi:http://dx.doi.org/10.17058/cinergis.v18i1.8160. 